\title{
A HIGH GAIN TAPERED SLOT VIVALDI ANTENNA BASED ON DIELECTRIC LENS AND CORRUGATED SLOTS
}

\author{
'Shams Khaled Ahmed'
}

\author{
Zaid A. Abdul Hassain ${ }^{2}$
}

Amer Abood Al-behadili ${ }^{3}$

1) Electrical Engineering, College of Engineering, Mustansiriyah University Baghdad, Iraq

2) Electrical Engineering, College of Engineering, Mustansiriyah University Baghdad, Iraq

3) Electrical Engineering, College of Engineering, Mustansiriyah University Baghdad, Iraq

\begin{abstract}
A gain improved Ultra-wideband (UWB) compact Vivaldi antenna for various applications such as radar and imaging applications with $41 \times 48 \times 1 \mathrm{~mm} 3$ size is designed. A periodic slit on the edges and a polygonshaped dielectric lens with directors are inserted to the tapered slot Vivaldi antenna to enhance the performances including small size, wide bandwidth, and high gain. The proposed Vivaldi antenna is executing from $(2.7-12) \mathrm{GHz}$ bandwidth. The gain is improved up to $5 \mathrm{~dB}$ at the lower range of frequency by clipping slit on the edge of the proposed antenna, however, more than $2 \mathrm{~dB}$ is the improvement of the gain at the center frequency. The proposed Vivaldi antenna shows approximate stable radiation patterns in the frequency range.
\end{abstract}

Keywords: End-fire, High directivity, Microwave imaging, Ultra-wideband, Vivaldi antenna

\section{Introduction}

Imaging and radar application demand including, stable radiation characteristics, wide bandwidth, and high gain $[1,2]$. Printed tapered slot Vivaldi antenna (TSVA) provides these demands [3] as, it has a faint profile, directional radiation pattern, and wide bandwidth. Yet, the extension of bandwidth, dimension diminishing, and enhancing the radiation characteristics of TSVA are complicated [4-9]. As presented in
[6] a modified VA with regular slot edge, loaded lens, and rectangular directors. The gain of the Vivaldi antenna is enhanced by the Inhomogeneously zero-index meta-material ZIM, and anisotropic IA ZIM has shown in [7]. In [8-14] corrugations are utilized to enhance the radiation features of the VA. In [15] a tapered slot edge construction is designed. A modified Vivaldi antenna is proposed In this paper the gain enhancement established by slotted edge patch, lens, and directors. The tapered slot Vivaldi antenna is designed and optimizes by (HFSS) Ansoft's High-Frequency Structure Simulator [16]. your findings with previous works or published results of other researchers. An additional section is allowed as long as the main section structure is intact.

\section{Antenna Structure And Design}

The proposed antenna configuration is shown in Fig. 1. The antenna of size $41 \times 48 \times 1 \mathrm{~mm}^{3}$ is stamped on an inexpensive FR4 substrate of $\varepsilon_{\mathrm{r}}$ $=4.4$. The designed tapered slot VA consists of an exponentially tapered slot, microstrip-to-slot line transmission construction, and a microstrip

*Corresponding Author: memememe.19961993@gmail.com 
feeding line on the other side. The flaring part is the essential part of the radiation of antennas for the entire frequency domain, and severance frequency is determined by the opening width. However, the expansion of the length obviously expanses the antenna size. Furthermore, the radiation fashion and the round cavity of the antenna define the essential manner and antenna features. On another face (back side) of the substrate, a linearly tapered microstrip line is approached. To provide excitation to the tapered slot Vivaldi antenna, a microstrip line to slot line transmission is modeled so the antenna still matched through a wide frequency domain.

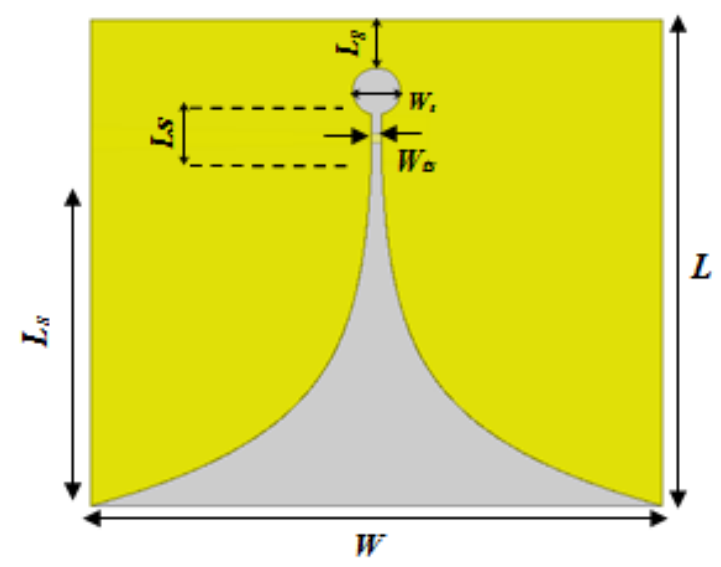

(a)

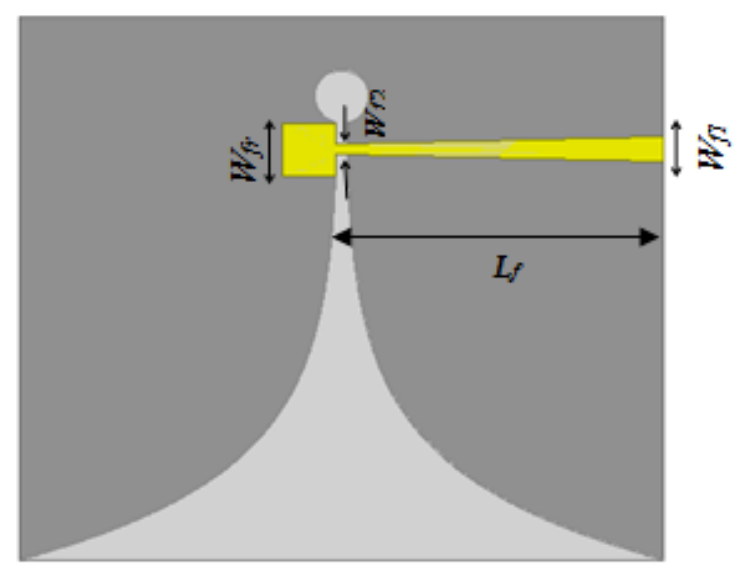

(b)

Figure 1. TSVA: (a) front view, and (b) back view.
The exponential tapered utilized in the suggested tapered slot Vivaldi antenna is given as:

$y(x)=9.19 e^{150 x}+0.134$

$11 \mathrm{~mm} \leq \mathrm{x} \leq 41 \mathrm{~mm}$

The following table Table 1 shows The designed tapered slot Vivaldi antenna parameters in $(\mathrm{mm})$.

\begin{tabular}{ccccccc}
\multicolumn{7}{c}{ Table 1. TSVA parameters in $(\mathrm{mm})$} \\
\hline$W$ & $L$ & $T$ & $L_{g}$ & $W_{s}$ & $W_{t s}$ & $L_{t s}$ \\
48 & 41 & 1 & 4 & 4 & 0.8 & 3 \\
\hline$L_{S}$ & $W_{f 1}$ & $W_{f 2}$ & $L_{f}$ & $W_{f r}$ & $R$ & \\
30 & 1.9 & 0.8 & 4 & 24.4 & 150 & \\
\hline
\end{tabular}

\section{Results And Analysis}

The designed tapered slot Vivaldi antenna is exciting with frequency domain from 2.-12 GHz is designed and simulated. To enhance the gain of the suggested tapered slot Vivaldi antenna Moreover, three kinds of improvement techniques are inserted. First, a polygonal dielectric lens is added at the radiation structure end of the TSVA, second, three rectangular mineral directors are printed on the lens aspect in the direction of the antenna axis, and third, tapered corrugations are added on the sides of the tapered patch.

\subsection{Modified Tapered Slot Vivaldi Antenna with Polygonal Lens and Directors}

To improve the gain at the upper range of frequency, lens and directors have been used. The half-elliptical-shaped lens is not efficient because energy is spreading off from opening, particularly at the upper range of frequency. Thus, the proper shape of the lens is substantial to provide effective focusing. Wherefore a polygonal shape dielectric lens is suggested. The lens is done by expanding the substrate with a polygonal frame that has a similar width of the 
substrate. The lens jobe is to focus the energy toward the opening center of the tapered slot Vivaldi antenna.

Furthermore, the directors are printed on the lens aspect of the TSVA, the metallic directors consisted of three various lengths, and several widths metallic rectangular printed in the direction of radiation, hold as directive elements, to support the radiation improvement in the end-fire orientation. The directors are utilized to raise the gain in the middle frequency. The designed tapered slot Vivaldi antenna with lens and directors is presented in Fig. 2.

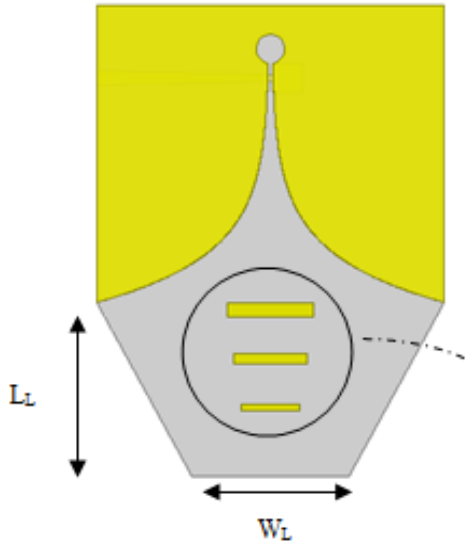

(a)

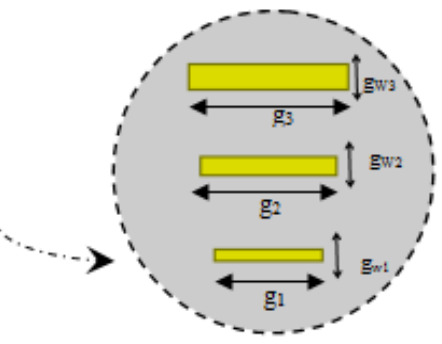

(b)
Figure 2. (a)TSVA with lens and directors, (b) directors' dimension

The dimensions of the suggested lens and three directors of the TSVA are listed in Table 2 in $\mathrm{mm}$.

Table 2. TSVA with lens and directors parameters in

\begin{tabular}{llllllll}
\multicolumn{8}{c}{$(\mathrm{mm})$} \\
\hline L1 & W1 & g1 & g2 & g3 & gw1 & gw2 & gw3 \\
24 & 21.8 & 8 & 10 & 12 & 1 & 1.5 & 2 \\
\hline
\end{tabular}

\subsubsection{The Reflection Coefficient}

The variations of the reflection coefficient S11 with frequency for the proposed tapered slot Vivaldi antenna, tapered slot Vivaldi antenna with the lens, and tapered slot Vivaldi antenna with lens and directors are shown in Fig. 3. It is observed that in the condition of TSVA $S_{11}$ less than $(-10) \mathrm{dB}$ for the domain $2.5-12 \mathrm{GHz}$. However, it is less than $(-10) \mathrm{dB}$ for the domain 2.6-12 GHz in the situation of TSVA with the lens. Whereas it is less $(-10) \mathrm{dB}$ for the domain 2.7-12 GHz in the condition the tapered slot Vivaldi antenna with lens and directors.

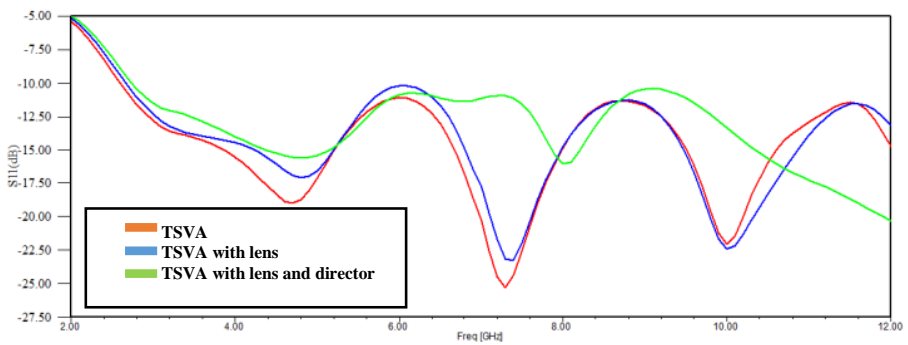

Figure 3. The reflection coefficient of the TSVA with lens and directors

\subsubsection{3-D Radiation Patterns}

The 3-D radiation patterns of the designed tapered slot Vivaldi antenna and tapered slot Vivaldi antenna with lens and TSVA with lens and directors for the frequencies $3 \mathrm{GHz}$, $6.77 \mathrm{GHz}, 10 \mathrm{GHz}$ are shown in Fig. 4. The designed TSVA with lens and the directors present steady end-fire radiation. It is observed that the radiation of the designed tapered slot Vivaldi antenna with lens and directors is extra directed and the side lobes are lower than, the designed TSVA and the suggested TSVA with the lens on.

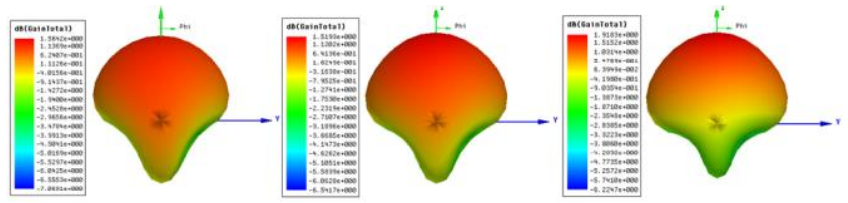

(a)

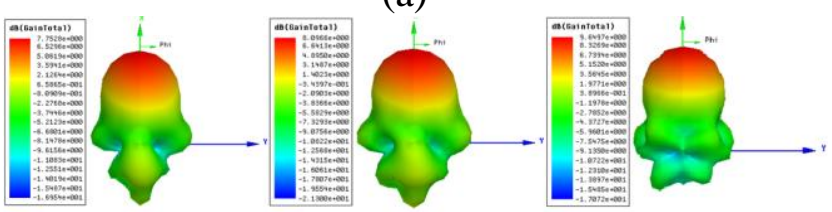

(b)

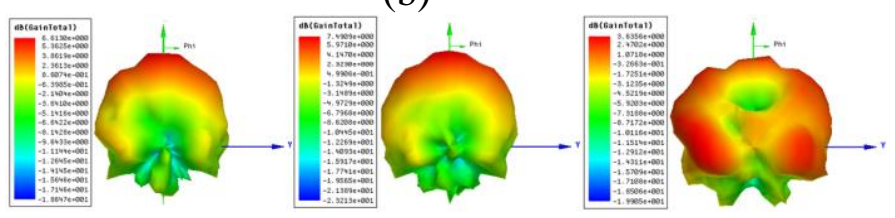

(c)

Figure 4. 3-D radiation pattern: (a) $3 \mathrm{GHz}$, (b) $6.77 \mathrm{GHz}$, (c) $10 \mathrm{GHz}$ 
The variation of the recognized gain of the proposed tapered slot Vivaldi antenna, tapered slot Vivaldi antenna with the lens, and tapered slot Vivaldi antenna with lens and directors is presented in Fig. 5. It is observed that due to the expansion of the substrate to create lens the gain of the designed tapered slot Vivaldi antenna improved extremely through the execution frequency domain. Furthermore, Due to the three rectangular directors, the gain enhancing is considerable in the middle frequency however it's quietly weak in the upper domain of the band.

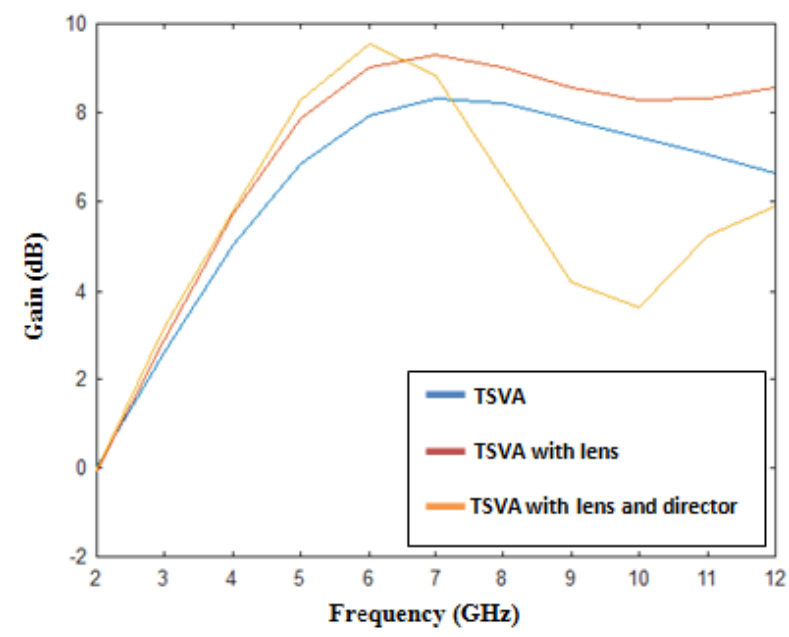

Figure 5. Gain variation with frequency of the TSVA with lens and directors

\subsection{Modified TSVA With Tapered corrugations}

The corrugations are molded by clipping different lengths of rectangular slots from the copper of the exponential tapered of both sides. The distance between the rectangular corrugations and the width of the slots are remaining the same. However, the lengths of the slots are variables. The loading of the corrugations on the edges of the exponentially tapered slot, act as a resistive loading and reducing the currents on the outward edges, By modifying the width and the length of the slot, the reactance of slot line can be modified, and improving the impedance matching, there for the performance of the antenna can be improving and maximum field remain concentrated across the flaring end and participate to the end-fire radiation patterns. The modified corrugated TSVA is shown in Fig. 6.

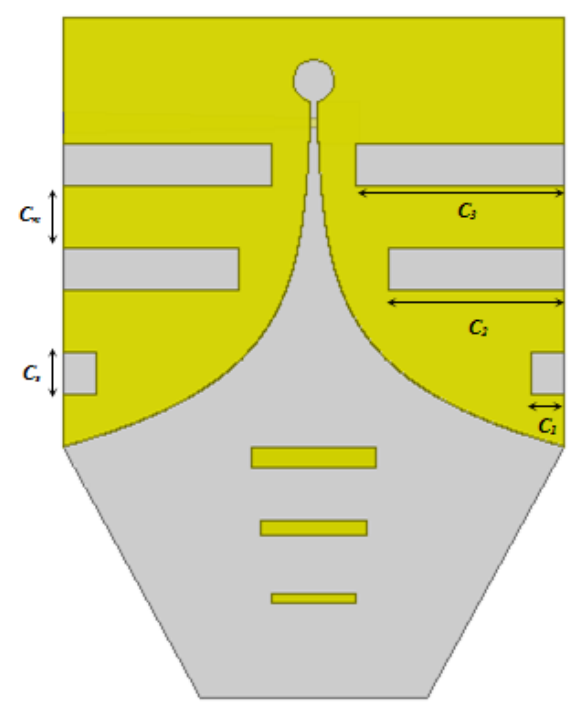

Figure 6. The proposed corrugated TSVA with polygon lens and directors

The suggested corrugated tapered TVA with polygonal lens and three mineral directors has slots dimension listed in Table $3 \mathrm{in} \mathrm{mm}$, the corrugated tapered slot Vivaldi antenna with lens and directors executed at domain 2.5-12 $\mathrm{GHz}$.

Table 3. Slots parameters in $(\mathrm{mm})$

\begin{tabular}{lllll}
\hline C1 & C2 & C3 & Cs & Cw \\
3.2 & 16.9 & 19.98 & 4 & 5 \\
\hline
\end{tabular}

The length of the corrugated slots was chosen according to the following equation:

$y(x)=9.2 \times 10^{-5} \times e^{150 x}+3.5 \times 10^{-3}$

\subsubsection{The Reflection Coefficient}

The variations of S11 with frequency for the proposed TSVA and the proposed corrugated tapered slot Vivaldi antenna with lens and directors are shown in Fig. 7. It is observed that the condition of the proposed Vivaldi Antenna $\mathrm{S}_{11}$ is less than (-10) $\mathrm{dB}$ for the domain 2.5-12 $\mathrm{GHz}$ which is similar to the condition of the corrugated TSVA with lens and directors. 


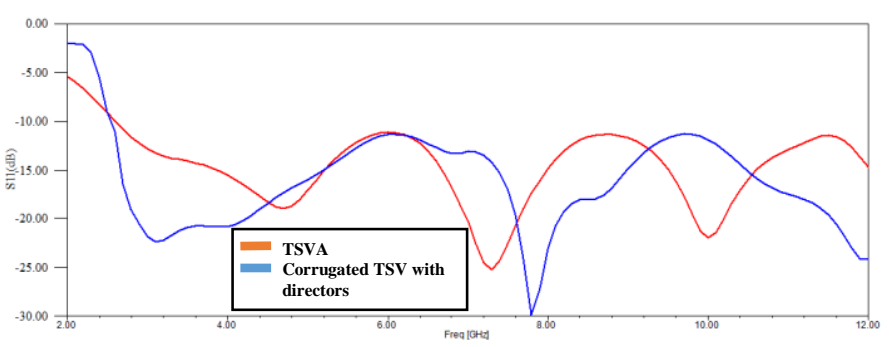

Figure 7. The reflection coefficient of corrugated TSVA with lens and directors

\subsubsection{3-D Radiation Patterns}

The 3-D radiation patterns of the designed tapered slot VA, and the designed corrugated tapered slot VA with lens and the directors for the following frequencies $3 \mathrm{GHz}, 6.77 \mathrm{GHz}$, and $10 \mathrm{GHz}$ are shown in Fig. 8. Both the proposed tapered slot Vivaldi antenna It's observed that the proposed corrugated TSVA with lens and the directors presents a stabilized end-fire radiation pattern in the low and center frequency.

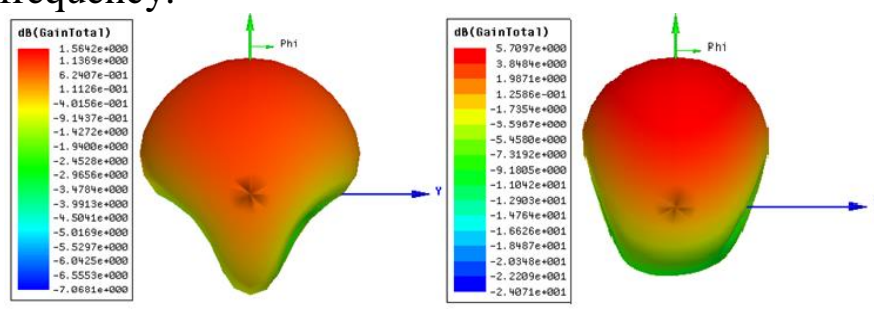

(a)

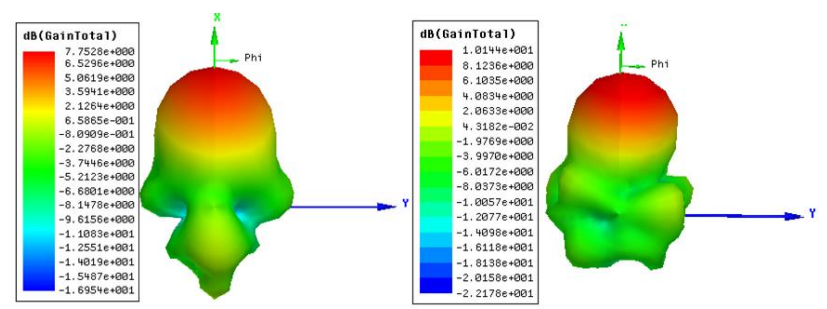

(b)

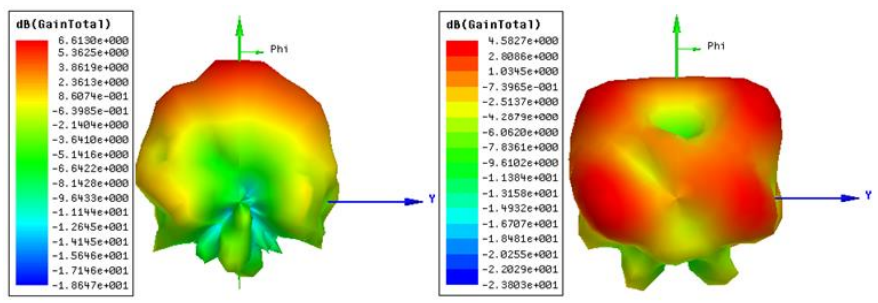

(c)

Figure 8. 3D The radiation pattern: (a) at $3 \mathrm{GHz}$, (b) at $6.77 \mathrm{GHz}$, (c) at $10 \mathrm{GHz}$
The variation of the perceived gain of the designed tapered slot VA, and the designed corrugated tapered slot VA with lens and directors is presented in Fig.9. It is observed that due to the expansion of the substrate to create a lens and the three directors and the slots made in the exponential tapered on the antenna sides the gain of the designed tapered slot Vivaldi antenna is improved extremely through the executing frequency domain. Due to the three rectangular slots, the gain enhanced magnificently in the lower band. Therefor The gain enhanced corrugated TSVA with lens and directors may be applied for applications like radars and microwave imaging.

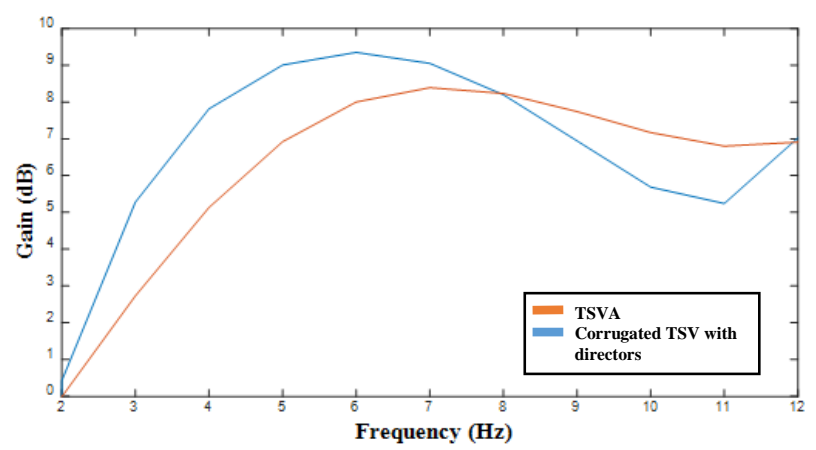

Figure 9. Gain variation with frequency of the corrugated TSVA with lens and directors

\section{Conclusion}

In this paper, a high gain corrugated TSVA with dielectric lens and three mineral directors has been suggested for imaging applications. The proposed Vivaldi antenna is intended with a periodic slit on the edge also the lens and directors are added to the compact UWB Vivaldi antenna. The designed tapered slot Vivaldi antenna affected by the techniques applied to enhance the gain and directivity of the proposed Vivaldi antenna, however, the antenna size is still compact as possible. The designed Vivaldi antenna is UWB and has a bandwidth of $2.7-12 \mathrm{GHz}$. Radiation patterns and the input of the proposed Vivaldi antennas have been plotted to realize the execution principles. 


\section{Conflict Of Interest}

The publication of this article causes no conflict of interest.

\section{References}

1. Song, Y., Y. C. Jiao, T. L. Zhang, G. Zhao, and F. S. Zhang, "Small tapered slot antenna with aband-notched function for wireless applications," Progress In Electromagnetics Research, Vol. 10, pp. 97-105, 2009.

2. Bai, J., S. Y. Shi, and D. W. Prather, "Modi ${ }^{-}$ed compact antipodal Vivaldi antenna for 4-50GHz UWB application," IEEE Trans. Microwave Theory Tech., vol. 59, no. 4, pp. 1051-1057, 2011.

3. M. Okoniewski, E. C. Fear, and Bourqui, J, (Balanced antipodal Vivaldi antenna with dielectric director for near-field microwave imaging)," IEEE Trans. Antennas Propagate"., 2010 , 2318-2326, Vol. 58, No. 7.

4. Y. Wang, A. E. Fathy, and Yang, Y., (Design of compact Vivaldi antenna arrays for UWB see through wall applications)," Progress In Electromagnetics Research", 2008, 401-418 ,Vol. 82..

5. A., K. M. Aghdam, R. F. Dana, and Mehdipour, (Completed dispersion analysis of Vivaldi antenna for ultra wideband applications)," Progress In Electromagnetics Research", 2007, Vol. 77, 85-96.

6. Taha A. Elwiand Zaid, A. Abed ALHussain, and Adham R. Azeez. "A Numerical Study of the Antipodal Vivaldi Antenna Design for Ultra Wideband Applications, " SAUSSUREA (ISSN: 03732525), vo. 7(1), pp. 12-16, 2017.

7. Zaid A. Abed AL-Husain, Amer A. Osman, and Sadiq K. Ahmed, "Design of UltraWideband Microstrip Antennas with Slots and Fractal Based Ground Plane, " Journal of
Al Rafidain University College, issue no. 34, 2014.

8. Lect.Zaid A. Abed AL-Husain, "Design of Microstrip Antennas Based on Fractal Geometry with Parasitic Patches for UltraWideband Applications, " Al-Mansour Journal, issue (21).

9. TJ. H. Qiu, P. Y. Zhang, eni, and G., N. Zhang, (Research on a novel miniaturized antipodal Vivaldi antenna with improved radiation)," IEEE Antennas and Wireless Propagat. Lett"., 2013, 417-420,Vol. 12.

10. W. Hu, F. S. Zhang, and Fei, P., Y. C. Jiao, (A miniaturized antipodal Vivaldi antenna with improved radiation characteristics)," IEEE Antennas and Wireless Propagat. Lett"., 2011, 127-130, Vol. 10.

11. H. Li, X. Y. Zou, T. J. Cui, and Zhou, B., (Broadband and high-gain planar Vivaldi antennas based on inhomogeneous anisotropic zero-index metamaterials )," Progress In Electromagnetics Research" , 2011, 235-247, Vol. 120.

12. J. Wang, L. X. Ma, Xu, H. Y., and H. Zhang, (A new tapered slot antenna with symmetrical and stable radiation pattern)," Progress In Electromagnetics Research Letters", 2008, 35-43, Vol. 5. Progress In Electromagnetics Research, 2014, Vol. 148.

13. Y. C. Jiao, L. Zhang, F. S. Zhang, Wang, N. B., and Y. Song, (Extreme wideband tapered slot antenna with impedance bandwidth in excess of 21:6) : 1," Journal of Electromagnetic Waves and Applications", 2009, 231-238, Vol. 23, Nos. 2-3.

14. M. E. Y. F. Wang, and Bialkowski, (A size-reduced exponentially tapered slot antenna with corrugations for directivity improvement)," Microwave Conference", 2009, 2482-2485, Singapore, 
15. P. J. Gibson, (The Vivaldi aerial), "in Proc. the 9th European Microwave Conference", 1979, pp. $101-105$.

16. Ansoft high frequency structure simulator (HFSS). [Online]. Available: www.ansys.com 